\title{
Paediatric anxiety disorders confer a considerable public health burden
}

By Dr. Jessica Edwards

Anxiety disorders usually begin in childhood or adolescence and are the most common mental health condition across the life span., ${ }^{1,2}$ Consequently, intense research efforts are focused on delineating the underlying mechanisms of paediatric anxiety so that we can better identify those at risk and intervene early. Earlier this year, Jeffrey Strawn and colleagues compiled a Research Review for the Journal of Child Psychology and Psychiatry discussing the substantial progress made over the past decade with regards to understanding the epidemiology, neurobiology and treatment of anxiety disorders in childhood and adolescence.

Strawn et al. first outline the epidemiology of specific phobia, separation anxiety disorder, social anxiety disorder, generalized anxiety disorder, and panic disorder highlighting the key characteristics, mean age of onset and prevalence. Then, they describe various risk factors for anxiety disorders - including cognitive, behavioural, family and wider environmental risk factors. They also discuss how some of these risk factors might be linked to neurobiology. 
Next, Strawn et al. give a clear and concise explanation of the neurobiology of anxiety disorders, drawing on findings made from structural and functional neuroimaging, neurocircuitry and neurochemistry analyses. They found from their literature review that structural and functional alterations in the frontolimbic system (including the amygdala, which is involved in fear processing) are consistently seen in young people with or at risk of developing anxiety disorders. But despite this knowledge, our understanding of developmental neurobiology and how it can predict risk of developing anxiety or treatment responses is still in its infancy.

Finally, Strawn and colleagues outline the results of dozens of studies that have investigated the efficacy of pharmacological and psychological treatment strategies. Many studies have demonstrated efficacy of various medications (including selective serotonin reuptake inhibitors) and psychotherapies (including cognitive behavioural therapy) available. What is lacking is an understanding of the best treatment combinations, the long-term treatment outcomes and the durability of response and remission. Furthermore, we don't yet have a clear picture of which patients will do best and with what treatment. Strawn et al. explain that these questions constitute essential areas for further research over the next decade.

"The last 5 years have seen an acceleration in research related to risk, neurobiology and treatment of anxiety disorders, which will almost certainly be translated into better, more effective and more personalized treatments for children and adolescents with anxiety disorders," says Strawn. "Until recently, anxiety was often underappreciated in terms of its impact, its developmental significance and its morbidity. For many, anxiety was contextualized as 'normal' or 'a phase'. Now, clinicians, policymakers, teachers and others working with youth increasingly appreciate its significance."

\section{Referring to:}

Strawn, J.R., Lu, L., Peris, T.S., Levine, A. \& Walkup, J.T. (2020), Research Review: Pediatric anxiety disorders - what have we learnt in the last 10 years? J. Child Psychol. Psychiatr. doi: 10.1111/ jcpp. 13262

\section{References:}

${ }^{1}$ Kessler RC, Berglund P, Demler O, Jin R, Merikangas KR, Walters $E E$. Lifetime prevalence and age-of-onset distributions of DSM-IV disorders in the National Comorbidity Survey Replication. Arch Gen Psychiatry 2005; 62: 593.

${ }^{2}$ Merikangas $\mathrm{KR}, \mathrm{He}$ J, Burstein $\mathrm{M}$, et al. Lifetime prevalence of mental disorders in US adolescents: results from the National Comorbidity Survey Replication-Adolescent Supplement (NCS-A). J Am Acad Child Adolesc Psychiatry 2010; 49: 980-9.

\section{Glossary:}

Cognitive behavioural therapy: a form of talking therapy that encourages patients to adapt the way they think and behave to improve the way they feel. CBT is based on the concept that thoughts, behaviour and feelings are interconnected. CBT tends to focus on current problems and finds practical ways to change negative pattens, in order to develop more helpful strategies of addressing these problems.

Generalized anxiety disorder: characterized by excessive diffuse anxiety, accompanied by difficulty sleeping, poor concentration, irritability, fatigue and muscle tension.

Selective serotonin reuptake inhibitors: a class of medication that is used in the treatment of anxiety and depressive disorders. These drugs increase serotonin levels in the brain by limiting its reuptake into cells. The effect is an increased level of serotonin available to bind with receptors.

Separation anxiety disorder: characterized by excessive distress experienced by a child when separated (or anticipating being separated) from their attachment figures. Therefore, the child usually seeks to be close to their attachment figures.

Social anxiety disorder: characterized by self-consciousness and intense anxiety in social situations, with worry or fear about being negatively judged or embarrassed. Consequently, the person often tries to escape from or avoid social situations.

Specific phobia: characterized by excessive fear of a particular situation or focus. As a result, the person often tries to escape from or avoid the feared situation/focus.

Panic disorder: characterized by panic attacks, which are discrete, rapid-onset and intense periods of distressing anxiety, including somatic symptoms (e.g. palpitations, feeling short of breath, or trembling) and cognitive symptoms (e.g. thoughts that "I'm going to die"). 